University of Nebraska - Lincoln

DigitalCommons@University of Nebraska - Lincoln

Robert Katz Publications

Research Papers in Physics and Astronomy

September 1980

Heavy Ion Beam Model for Radiobiology

Rose Ann Roth

IBM Corp., Essex Junction, VT

Robert Katz

University of Nebraska-Lincoln, rkatz2@unl.edu

Follow this and additional works at: https://digitalcommons.unl.edu/physicskatz

Part of the Physics Commons

Roth, Rose Ann and Katz, Robert, "Heavy Ion Beam Model for Radiobiology" (1980). Robert Katz

Publications. 31.

https://digitalcommons.unl.edu/physicskatz/31

This Article is brought to you for free and open access by the Research Papers in Physics and Astronomy at DigitalCommons@University of Nebraska - Lincoln. It has been accepted for inclusion in Robert Katz Publications by an authorized administrator of DigitalCommons@University of Nebraska - Lincoln. 
Published in Radiation Research 83:3 (September 1980), pp. 499-510. Published by the Radiation Research Society; copyright (C) 1980 by Academic Press, Inc. Used by permission.

Submitted August 17, 1979; revised February 25, 1980.

Research supported by the U. S. Department of Energy.

\title{
Heavy Ion Beam Model for Radiobiology
}

\author{
Rose Ann Roth* and Robert Katz \\ University of Nebraska-Lincoln, Lincoln, Nebraska 68588 \\ * Present address: R. A. (Roth) Krauter, IBM Corp., Essex Junction, VT 05452.
}

\begin{abstract}
An ad hoc model of energetic heavy ion beams, including secondary and tertiary particles, has been constructed for predicting radiobiological experiments. While the beam model is relatively primitive, it yields depth-dose and depth-radiobiological calculations in good agreement with experiment upstream of the Bragg peak. Beyond the peak, the model is somewhat coarse grained and seems to underestimate low-LET fragment production. These defects can be repaired at some cost in computer time. Presently a complete set of depth-dose and radiobiological results (RBE, OER, aerobic and hypoxic survival) is obtained in $4-8 \mathrm{~min}$, for a single beam, at a cost of $\$ 10$. The model can be extended to mixed radiation fields, or to explore the design of ridge filters. These predictions are based on cellular radiosensitivity parameters extracted from track-segment irradiations at about $8 \mathrm{MeV} / \mathrm{amu}$. Their success implies that no new radiobiological results arise from irradiation with beams at $500 \mathrm{MeV} / \mathrm{amu}$.
\end{abstract}

\section{Introduction}

Recent dosimetric and radiobiological studies with heavy ion beams (1-4) have prompted us to extend an earlier beam model $(5,6)$ to include the propagation of secondary and tertiary particles. We have been able to construct an ad hoc model of a particle beam which yields good agreement with both dose and radiobiological measurements upstream of the Bragg peak. The disagreement beyond the Bragg peak seems repairable, as more accurate knowledge of interaction cross sections is included in the model, and the computational steps are made finer grained, but these refinements may require greatly extended computation time and expense and are presently beyond our budgetary constraints. The present beam model suffices to exhibit the remarkable degree to which the theory of $\mathrm{RBE}$ can predict present radiobiological findings from formulas and radiosensitivity parameters established almost a decade ago (7).

\section{Theory of Biological Effects}

The details of the $\delta$-ray theory of track structure as applied to biological cells are given elsewhere (5-7). To refresh the reader, the theory applies four experimental radiosensitivity parameters to a collection of formulas which yield predictions of cellular survival. The parameters are $m$ and $E_{0}$, the extrapolation number and extrapolated D-37 dose 
extracted from experimental survival curves determined with $\gamma$ rays, and $\sigma_{0}$ and $\kappa$, the plateau value of the cross section and the value of $z^{2} / 4 \beta^{2}$ of the track-segment irradiation with heavy ions at which it is achieved. Here $z$ is the effective charge number and $\beta$ is the relative speed of the bombarding ion.

The probability that a single ion passing through the cell inactivates it is given by the expression

$$
P=\left[1-\exp \left(-z^{2} / \kappa \beta^{2}\right)\right]^{m}
$$

with the effective charge number calculated from the atomic number $Z$ as

$$
z=Z\left[1-\exp \left(-125 \beta Z^{-2 / 3}\right)\right]
$$

Inactivation by single ions is called the "ion-kill" mode.

Cells are also inactivated by the accumulation of sublethal damage over the irradiation period from ions or their $\delta$ rays which intersect the cell nucleus. The equivalent $\gamma$-ray dose delivered by the ion beam is called $D_{\gamma}$. It depends on both the total dose $D$ of heavy ions and the probability $P$ for ion-kill inactivation (and thus on cellular parameters). It is given by

$$
D_{\gamma}=(1-P) D \text {. }
$$

In the ion-kill mode the cell killing depends on the fluence $F$ of the beam, and in the $\gamma$-kill mode it depends on the dose $D=F L$, where $L$ is $\mathrm{LET}_{\infty}$.

The fraction of cells surviving an irradiation is given as the product of the survival probabilities in the ion-kill and the $\gamma$-kill modes, represented by $\Pi_{i}$ and $\Pi_{\gamma}$, respectively. We have

$$
N / N_{0}=\Pi_{i} \times \Pi_{\gamma}
$$

with

$$
\begin{aligned}
& \Pi_{i}=\exp (-\sigma F), \\
& \Pi_{\gamma}=1-\left[1-\exp \left(-D_{\gamma} / E_{0}\right)\right]^{\mathrm{m}},
\end{aligned}
$$

and

$$
\sigma=\sigma_{0} P
$$

where we restrict irradiations to particles of such low $Z$ and $\beta$ that there is negligible probability that cells will be inactivated in the "track-width regime." For mixed radiation fields, we write

and

$$
\begin{aligned}
& D=\sum_{j} \sum_{k}\left(F_{j k} L_{j k}\right), \\
& D_{\gamma}=\sum_{j} \sum_{k}\left[F_{j k} L_{j k}\left(1-P_{j k}\right)\right],
\end{aligned}
$$

$$
\Pi_{i}=\exp -\left[\sum_{j} \sum_{k}\left(\sigma_{j k} F_{j k}\right)\right]
$$

In these expressions the subscripts designate the particles of atomic number $Z_{j}$, mass number $A_{j}$, and speed $\beta_{k}$. The quantity $\Pi_{\gamma}$ is found by substituting from Equation (9) into Equation (6). 


\section{Beam Model}

As in our earlier beam model (5), the primary beam is assumed to propagate without scattering, being attenuated with a partial interaction cross section $\sigma_{\mathrm{i}}$, given by $(8)$

$$
\sigma_{\mathrm{i}}=\pi\left(1.13 \times 10^{-13} \mathrm{~cm}\right)^{2}\left(A_{\text {projectile }}^{1 / 3}+A_{\mathrm{i}}^{1 / 3}\right)^{2} .
$$

Taking all target nuclei in the medium into account yields

$$
F_{x}=F_{0} \exp \left[-\left(\sum_{i=1}^{j} \sigma_{\mathrm{i}} \rho_{\mathrm{i}}\right) x\right],
$$

where $F_{x}$ is the fluence of primary particles at depth $x$ when $F_{0}$ is the incident fluence onto a medium containing $j$ nuclear species of number density $\rho$. This interaction cross section is apportioned to peripheral collisions, lumped into three sets of product particle pairs, whose relative intensity is estimated from limited experimental data (9). For the calculations made here $50 \%$ of the peripheral collisions are taken to result in a proton, a neutron (subsequently neglected), and a residual fragment of charge number $Z-1$ and mass number $A-2 ; 25 \%$ produce a He nucleus and a fragment $Z-2$ and $A-4 ; 8 \%$ yield a fragment of $Z=3$ and $A=6$ and a residual heavy fragment of $Z-3$ and $A-6$. All fragments are taken to move forward with the speed of the incident projectile at the point of collision. All other possible fragments from peripheral collisions, from central collisions, and from fragmentation of the target nucleus are ignored. Straggling of the primary beam, but not of the secondary particles, is accommodated by taking the incident monoenergetic beam to be made up of fractions whose different velocity-stopping power relations are constructed to yield the straggling Gaussian (10). In this construction, the stopping power and range of the central component of the Gaussian is based on tabulated proton stopping powers and ranges. In the energy interval from 0.1 to $2 \mathrm{MeV}$ we have used the table of Janni (11), for 2 to $10 \mathrm{MeV}$ the table of Northcliffe and Schilling (12), and from 10 to $5000 \mathrm{MeV}$ the table of Barkas and Berger (13). Stopping power and ranges for heavy ions are constructed from the proton values using expressions from Barkas and Berger, given here for completeness as follows:

$$
\begin{aligned}
& L(z, \beta)=L(p, \beta)\left[z(\beta) / z_{\mathrm{p}}(\beta)\right]^{2}, \\
& R_{\mathrm{i}}(\beta)=\left(A_{\mathrm{i}} / z^{2}\right)\left[R_{\mathrm{p}}(\beta)+B_{z}(\beta)\right] .
\end{aligned}
$$

Here $z$ and $z_{\mathrm{p}}$ are the effective charge numbers of the ion and proton, respectively, from Equation (2), calculated at relative speed $\beta ; L$ is the stopping power $\left(\mathrm{LET}_{\infty}\right) ; R_{\mathrm{i}}$ and $R_{\mathrm{p}}$ are the ion and proton ranges, respectively; and $B_{z}$ is a range correction factor given by

$$
\begin{array}{lll}
B_{z}(\beta): & \beta<2 Z / 137, & B_{z}=\beta Z^{5 / 3} C_{1}, \\
& \beta \geq 2 Z / 137, & B_{z}=Z^{8 / 3} C_{2} .
\end{array}
$$

For water, $C_{1}=2.28 \times 10^{-3} \mathrm{~g} / \mathrm{cm}^{2}$ and $C_{2}=3.34 \times 10^{-5} \mathrm{~g} / \mathrm{cm}^{2}$. In this model the stopping power and range are for water, while attenuation is for the nuclear composition of the medium. 
To keep track of all secondary and tertiary particles down the beam a table of 209 values of $\beta$, from 0.015 to 0.99 , is created in the computer along with corresponding tables of fluence for each secondary and tertiary particle type. The beam calculation is made by progressing from the incident energy to the stopping end. In each depth increment, the decrement in fluence produces an increment of next-generation particles. These are added to the appropriate "bins," which represent the fluence contribution and speed which these particles would have had if they were all incident at the surface of the absorber. All particles in a particular bin are then propagated, without straggling, by applying the same routine as is used for the central component of the primary beam. Thus secondaries generate tertiaries by the same rule which we use for the generation of secondaries by primaries. The use of this table introduces a structure in the depth-dose curves which is all too evident beyond the Bragg peak. This structure can be reduced by the use of more bins; a larger and more detailed array of peripheral interactions can be introduced to yield a better distribution of secondary fragments.

The computer program for the beam incorporates the theory of cellular survival $(5-7)$.

In its present form, the program uses a maximum core of $160 \mathrm{~K}$ and has a run time of 4-8 min of CPU time on an IBM 360/65J computer. One run of the program yields the following information: dose and surviving fraction of cells for up to 10 specified incident fluences along with RBE and OER for up to three specified survival levels at up to 400 depths in a water phantom. Calculations are made at specified positions or at centimeter steps in the plateau region and at appropriately finer steps in the region around the Bragg peak. For ridge filter calculations millimeter depth increments are used.

\section{Results-Dosimetry}

Depth-dose curves have been calculated for He, C, O, Ne, and Ar beams for comparison with published data of Maccabee and Ritter (O at $233 \mathrm{MeV} / \mathrm{amu}$ ) (14), Bridier and Fache (He at $162 \mathrm{MeV} / \mathrm{amu}$ ) (4), and Lyman and Howard (He at 150 and $225 \mathrm{MeV} /$ amu, $\mathrm{C}$ at 250 and $400 \mathrm{MeV} / \mathrm{amu}, \mathrm{Ne}$ at 400 and $594 \mathrm{MeV} / \mathrm{amu}$, and $\mathrm{Ar}$ at 500 and 900 $\mathrm{MeV} / \mathrm{amu}$ ) (1). Several of these results are displayed in Figures 1-4 for $\mathrm{He}, \mathrm{C}, \mathrm{Ne}$, and $\mathrm{Ar}$ beams at the lower energies which penetrate some 10 to $15 \mathrm{~cm}$ in the water phantom according to the experimental work of Lyman and Howard. In all cases the calculated dose at the Bragg peak is higher than the experimental value, as befits the difference between the monoenergetic calculated beam and the somewhat broader energy distribution of the experimental beam. In all cases the calculated curves display a computational structure beyond the Bragg peak that has already been discussed. We display the dose from the primary beam alone, labeled primary; the dose from the primary and the secondary fragments, labeled secondary; and the dose from the primary beam and the secondary and tertiary fragments, labeled tertiary. For the He beam no tertiary fragmentations are permitted by our rules, and so only the primary and secondary contributions are shown. In all cases the energy of the incident beam used in the calculation is adjusted to place the Bragg peak at the correct experimentally determined depth. We have not attempted to sort out the discrepancies between the energies given for the measured and the calculated beams, be- 


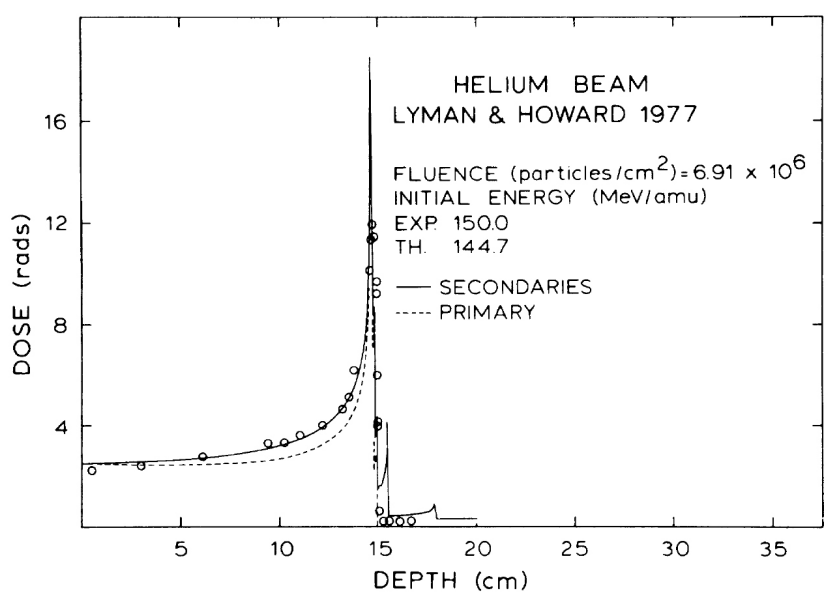

Figure 1. Calculated dose as a function of depth compared to experimental data (open circles) for a $150 \mathrm{MeV} / \mathrm{amu} \mathrm{He}$ beam. In this figure, as in Figures 2-4, the fluence chosen for the calculation yields best fit to the overall experimental data. The energy chosen for the calculated beam yields a calculated Bragg peak depth equal to the experimental depth. The quoted energy of the experimental beam is from measurements made upstream of the absorber. We show a dashed curve for the primary beam alone and a light line for the dose from primaries and secondaries. In subsequent curves, where applicable, a heavy line shows the dose from primaries, secondaries, and tertiaries. The jagged structure beyond the calculated Bragg peak arises from limitations in the beam model, discussed in the text.

lieving them to be due to the experimental conditions under which the measurements are made. The calculated beam is always incident on the absorber monoenergetically at the stated energy.

Our calculated depth-dose curves are in good agreement with the measured data upstream of the Bragg peak in both the displayed curves and the other calculated results

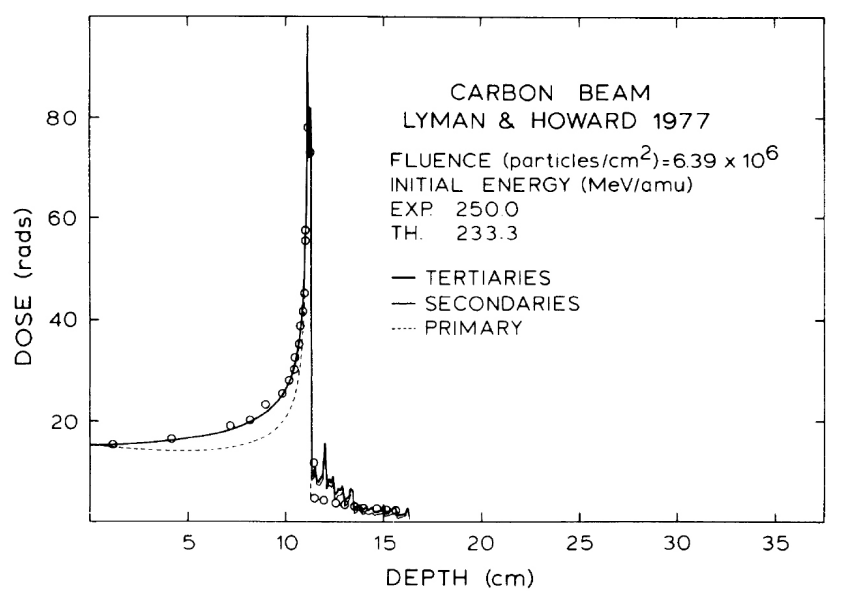

Figure 2. Carbon beam at $250 \mathrm{MeV} / \mathrm{amu}$. See caption to Figure 1. 


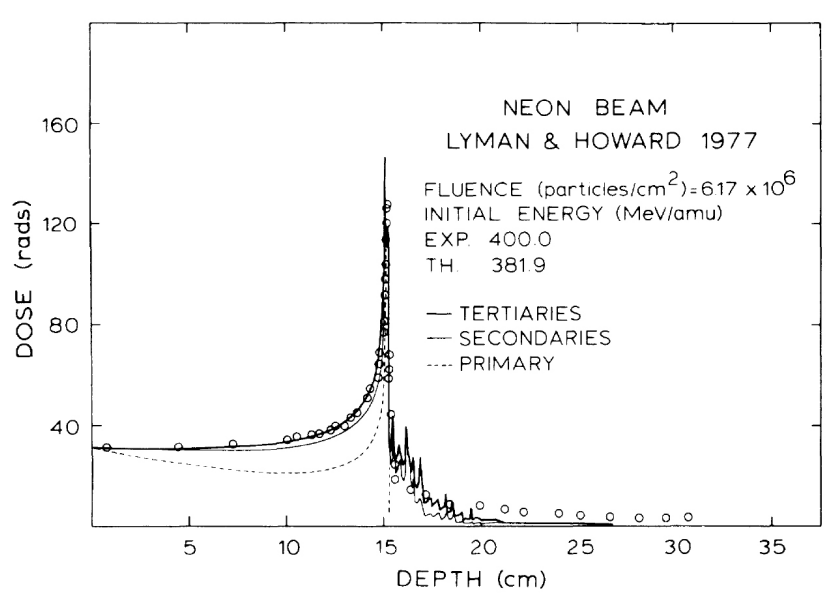

Figure 3. Neon beam at $400 \mathrm{MeV} / \mathrm{amu}$. See caption to Figure 1.

not displayed here. For the radiobiological calculations, the depth-dose curves represent a constraint which must be reasonably fitted by the beam model. The purpose of the model is to provide some approximation to the particle-energy spectrum as a function of depth in the beam, not presently available from experiment, or from other models known to us. According to track theory the particle-energy spectrum is required for radiobiological predictions. Neither the spectrum of LET values or of $z^{2} / \beta^{2}$ nor their averages can be expected to yield satisfactory radiobiological predictions.

\section{Results-Radiobiology}

Survival curves for aerobically and hypoxically irradiated T-1 human kidney cells as well as the RBE and OER at 10\% survival have been calculated as a function of depth in a phantom for $\mathrm{C}, \mathrm{Ne}$, and $\mathrm{Ar}$ beams. These are compared to the experimental data of Blake-

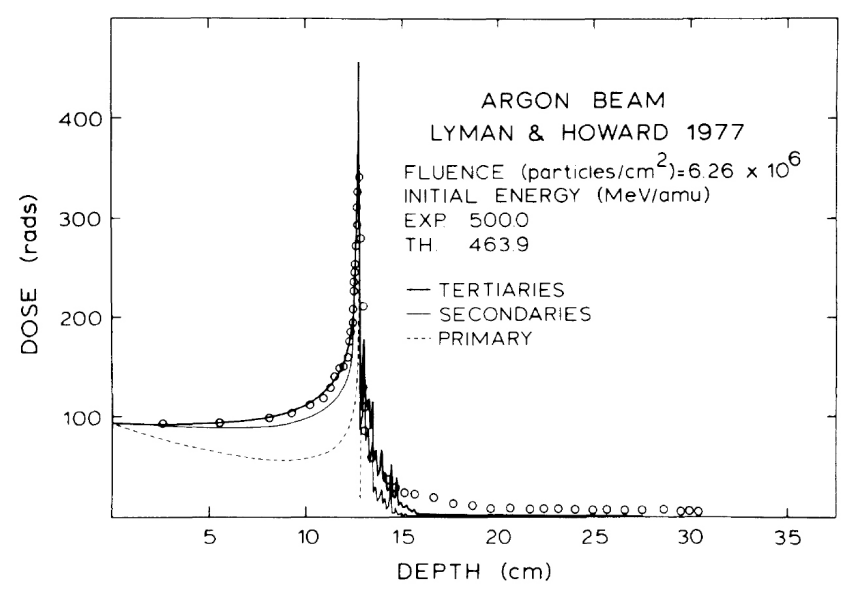

Figure 4. Argon beam at $500 \mathrm{MeV} / \mathrm{amu}$. See caption to Figure 1. 


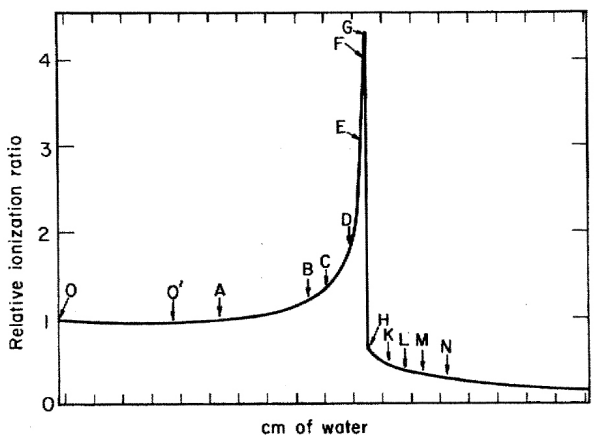

Figure 5. Schematic representation of depth-dose curves, giving the nominal positions at which radiobiological measurements were made (3), and for which theoretical radiobiological curves were calculated.

\section{CARBON}

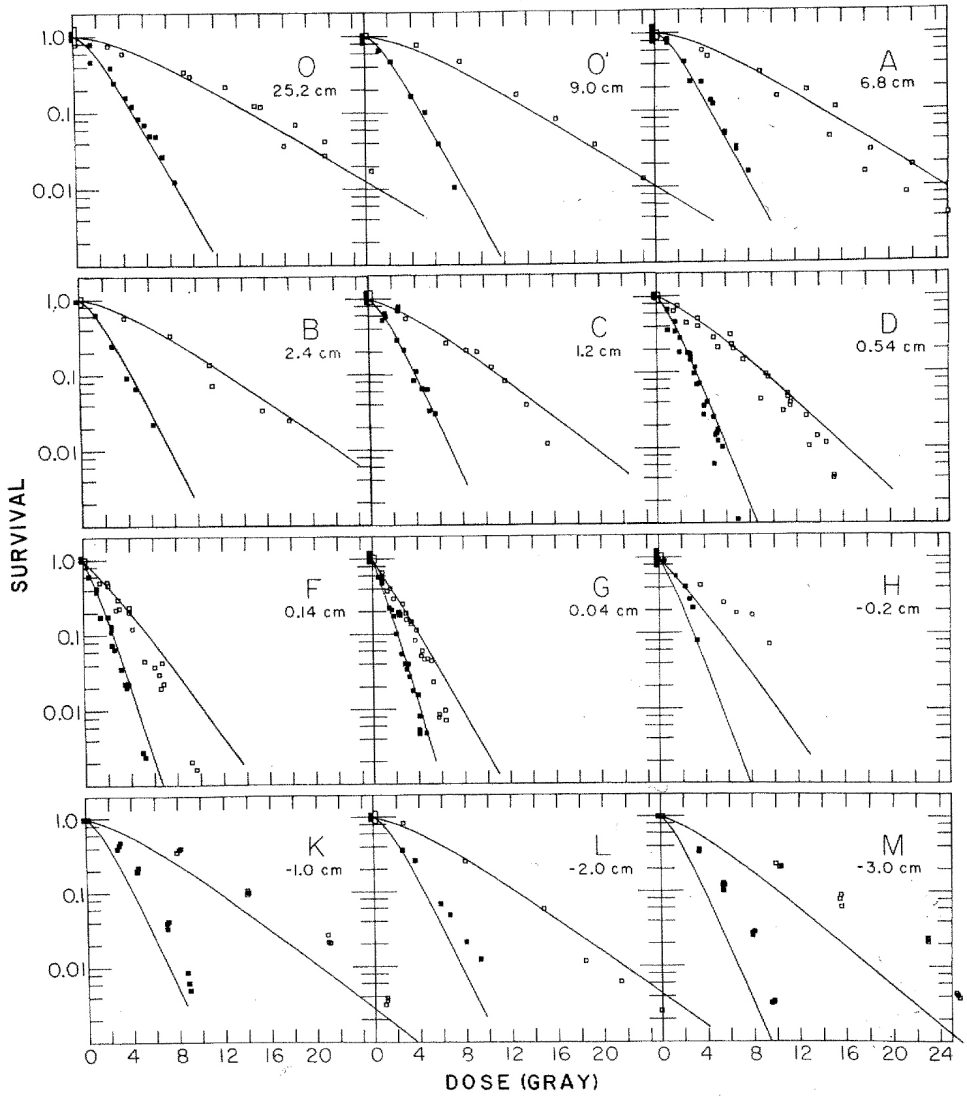

Figure 6. Survival curves for T-1 human kidney cells in a carbon beam at initial energy $400 \mathrm{MeV} /$ amu at the depths and nominal positions indicated in Figure 5. The data (3) for hypoxic cells are shown as open squares, while the data for aerobically irradiated cells are shown as solid squares. The lines are calculated from the theory and the beam model. 


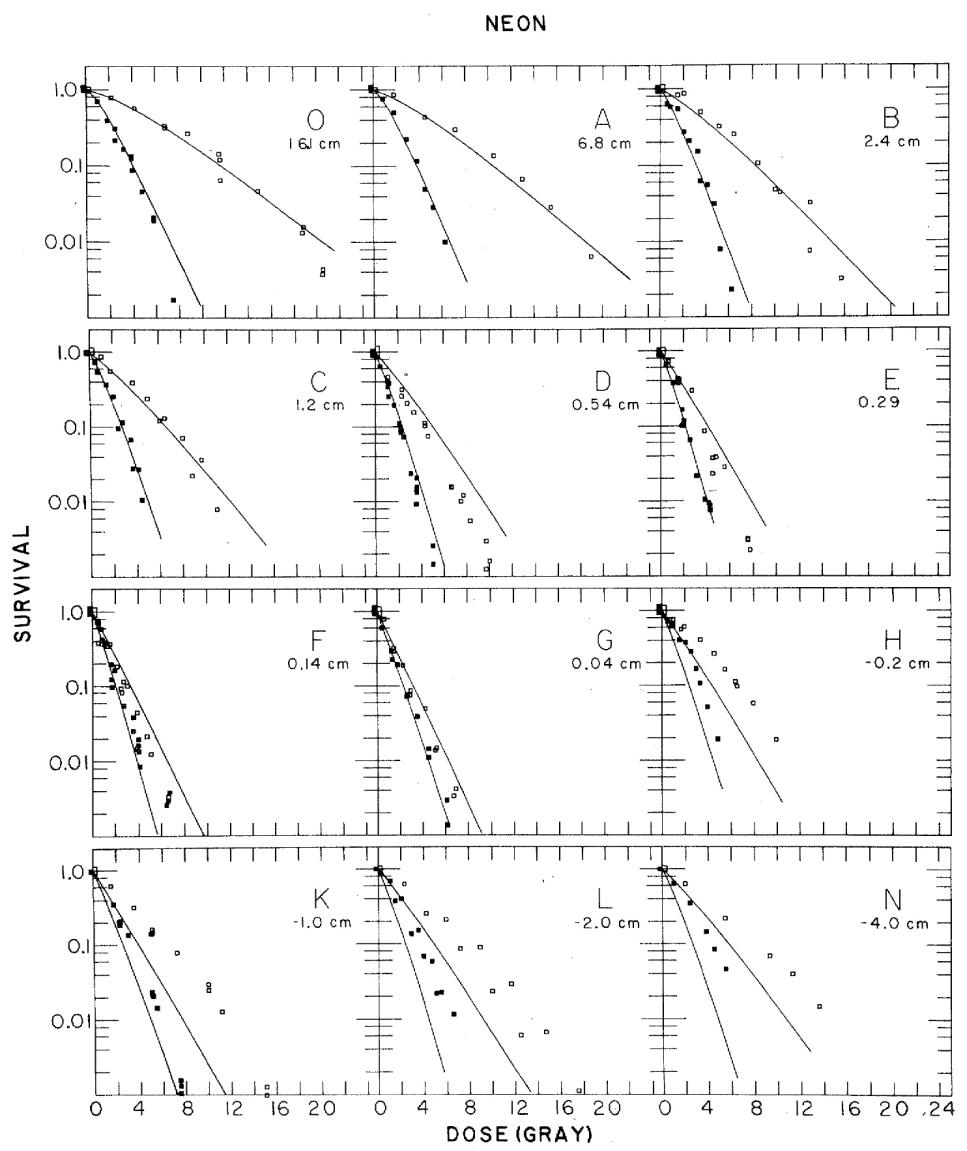

Figure 7. Survival curves as a function of depth in a neon beam of initial energy $425 \mathrm{MeV} / \mathrm{amu}$.

ley et al. (3). From their work we show in Figure 5 the nominal location of the positions in the beam at which their measurements were made for the identification of our subsequent survival curves.

For aerobically irradiated cells we have used the radiosensitivity parameters: $E_{0}=1.7$ $\times 10^{4} \mathrm{erg} / \mathrm{cm}^{3}, m=2.5, \kappa=1000$, and $\sigma_{0}=6.7 \times 10^{-7} \mathrm{~cm}^{2}$, unchanged from the visually fitted parameters (7) found from the experimental data of Todd (15) almost a decade ago. There the parameters for hypoxic cells were $E_{0}=4.6 \times 10^{4} \mathrm{erg} / \mathrm{cm}^{3}$ and $\kappa=1300$, with $\mathrm{m}$ and $\sigma_{0}$ as in the aerobic case. In the present experiments a higher degree of hypoxia was achieved, necessitating an adjustment of these hypoxic cell parameters of about $12 \%$ upward to match the observed OER at the plateau (0) position of the carbon beam of 3 . The values used in these calculations are $E_{0}=5.2 \times 10^{4} \mathrm{erg} / \mathrm{cm}^{3}$ and $\kappa=1450$. No other adjustments or fittings have been made.

In Figures 6-8 we show the calculated survival curves at the indicated positions as solid lines with the data of Blakeley et al. as squares. Closed squares are for aerobically irradiated kidney cells, while open squares are used for hypoxically irradiated cells. Dis- 


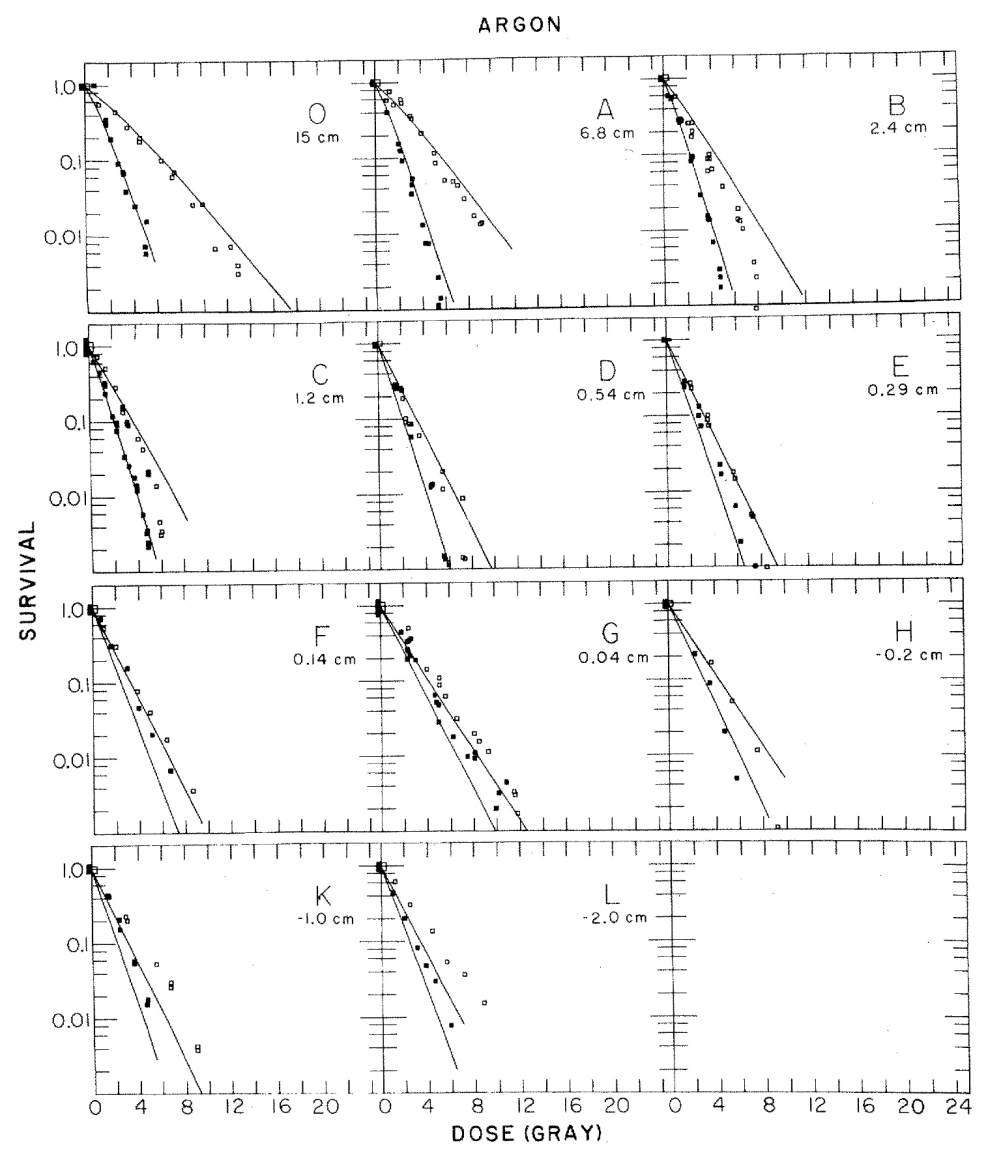

Figure 8. Survival curves as a function of depth in an argon beam of initial energy $570 \mathrm{MeV} / \mathrm{amu}$.

tances upstream of the Bragg peak are shown as positive, while distances downstream of the peak are shown as negative. The calculated survival curves for aerobically irradiated cells are in remarkable agreement with experiment upstream of the Bragg peak, except for positions E, F, and $\mathrm{G}$ in the Ar beam, within $3 \mathrm{~mm}$ of the Bragg peak. The results for hypoxic cells are somewhat more erratic. While the agreement between theory and experiment is quite good in most cases to $10 \%$ survival and perhaps somewhat lower, there is a clear tendency for the hypoxic cell data to display lower than the predicted survival at high doses. Here we must consider the possibility that the discrepancy is due to experimental problems and ask whether hypoxia has been consistently maintained in all experiments over all irradiations. It is difficult to understand why the theory might be in excellent agreement with the data for aerobic cells over more than two decades of survival but that it should disagree with experiment for hypoxic cells. Beyond the Bragg peak in all cases the calculated survival curves lie beneath those of the experimental data. This is attributed to a failure of the beam model in this region. Evidently the present beam model underestimates the low LET contribution from the secondary fragments. 

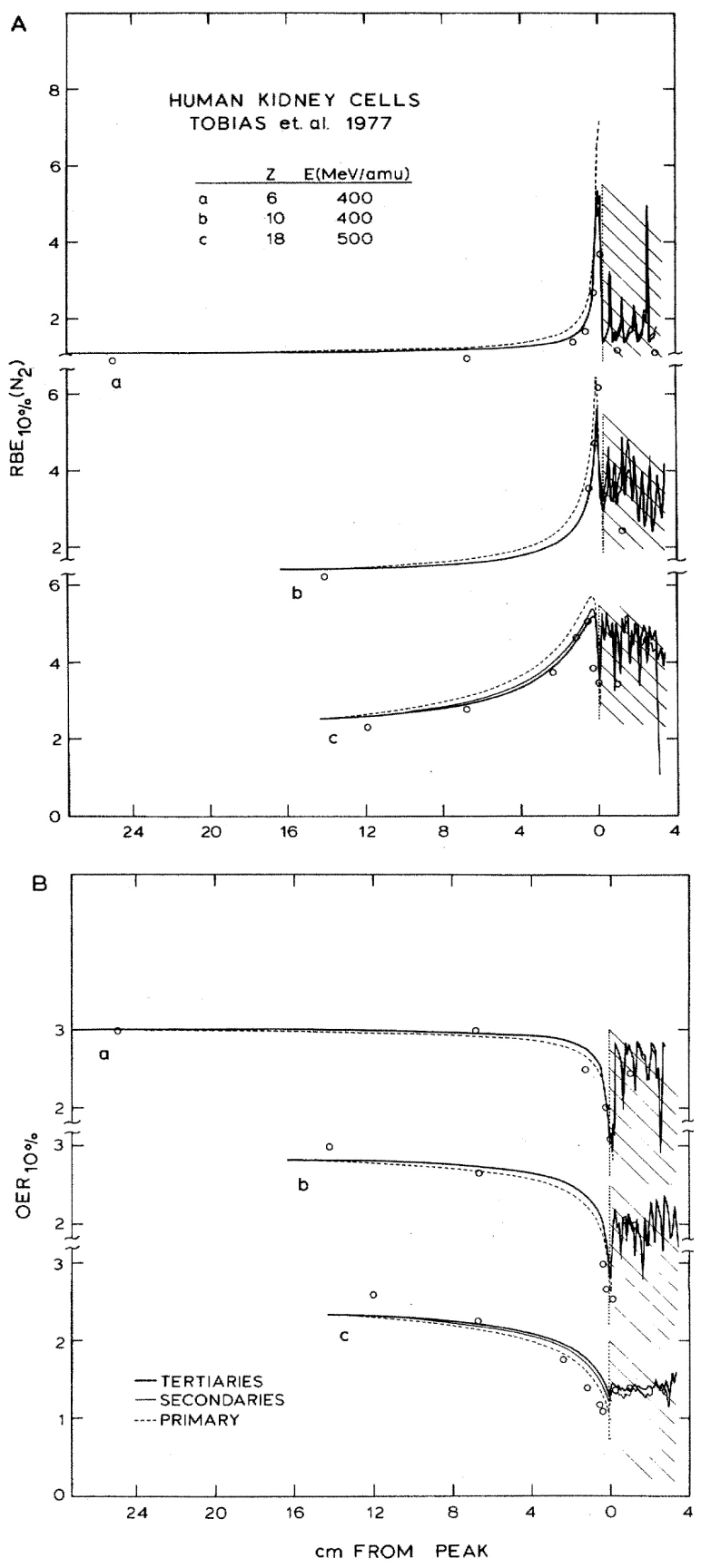

Figure 9. A.) Calculated values of the RBE of hypoxically irradiated cells are shown as a function of depth for energetic C, Ar, and Ne beams, in comparisons with the experimental data (2), shown as open circles. B.) Calculated values of the OER at $10 \%$ survival for human kidney cells are shown as a function of depth in these beams in comparison with the experimental data. For both Figures 9A \& $\mathrm{B}$, a cross-hatched region is shown beyond the Bragg peak, where the jagged character of the beam model calculations, arising from our grouping of the secondary particles, tends to distort the results. 
Calculations of the RBE of hypoxically irradiated cells and the OER, both at $10 \%$ survival, are compared to experimental data in Figures 9A and B. The comparison of these predictions with the data represents a weaker test of the theory than the survival curves themselves. The results beyond the Bragg peak are here cross-hatched since the structure of the beam model tends to distort the results in this region. Upstream of the Bragg peak both the RBE and OER data agree well with the theory. We see that the presence of secondary and tertiary additions to the primary beam do not have a great effect on either the RBE or the OER at $10 \%$ survival.

All in all the agreement between prediction and data is quite gratifying and lends support to the use of the theory in equipment design, or for whatever pretherapeutic assistance radiobiological experiments with kidney cells might provide. In its present form the theory inherently encompasses the effects of mixed radiation fields in the present calculation as well as in earlier calculations for pions and neutrons admixed with low-LET contaminants $(16,17)$.

\section{Ridge Filters-Design and Implications}

In earlier work with a beam model which did not generate secondary and tertiary particles, we explored ridge filters 5 and $10 \mathrm{~cm}$ wide $(5,6)$. There we found that single-port irradiation with heavy ion beams was inherently difficult to manage. One could not produce isoeffect regions with the same filter for both aerobic and hypoxic cells simultaneously. The OER varied widely across the irradiated volume. These results suggested that complications might ensue from single-port irradiations with high-LET irradiations, as with heavy ions or even with pions. Symmetric double-port irradiations were clearly to be preferred.

These conclusions are unaltered in the present work.

It appears to be relatively easy to design a symmetric double-port irradiation that will yield an isoeffect volume, while the difficulties with single-port irradiation remain. The addition of secondary and tertiary particles to the beam model tends to smooth out some of the irregularities in the depth-dose and the depth-effect relationships with the ridge filter, and a somewhat different design is required with secondary particles than with primary particles alone.

However, since in real therapy situations it cannot be routinely possible to have symmetric double-port irradiations, it appears that ridge filter design must be tailored to each case. In this event, it will be essential to obtain from therapists their best estimate of the radiosensitivity parameters of both normal tissue and diseased tissue so that ridge filters may be designed to the patient's needs.

\section{References}

1. J. T. Lyman and J. Howard, Biomedical research facilities and dosimetry. In Biological and Medical Research with Accelerated Heavy Ions at the Bevalac 1974-1977 (S. Elam, Ed.), pp. 26-35. Lawrence Berkeley Laboratory, University of California, Berkeley, 1977. [LBL-5610.]

2. C. A. Tobias, E. A. Blakeley, and R. J. Roots, Cellular and molecular biology. In Biological and Medical Research with Accelerated Heavy Ions at the Bevalac 1974-1977 (S. Elam, 
Ed.), pp. 76-110. Lawrence Berkeley Laboratory, University of California, Berkeley, 1977. [LBL-5610.]

3. E. A. Blakeley, C. A. Tobias, T. C. H. Yang, K. C. Smith, and J. T. Lyman, Inactivation of human kidney cells by high-energy monoenergetic heavy-ion beams. Radiat. Res. 80, 122-160 (1979). [See also Report LBL-5674, Lawrence Berkeley Laboratory, University of California, Berkeley (same title and authors) for tabulated survival data.]

4. A. Bridier and P. Fache, Etudes dosimetriques et microdosimetriques de faisceaux de particules a transfert lineique d'energie eleve. Doctoral thesis presented to the University Paul Sabatier of Toulouse, France, 1974. [Available as Report No. CEA-R-4655, Service de Documentation, CEN-Saclay, B. P. 2, 91190 Gif-sur-Yvette, France.]

5. R. Katz and S. C. Sharma, Response of cells to fast neutrons, stopped pions, and heavy ion beams. Nucl. Instrum. Meth. 111, 93-116 (1973).

6. R. Katz and S. C. Sharma, Heavy particles in therapy: An application of track theory. Phys. Med. Biol. 19, 413-435 (1974).

7. R. Katz, B. Ackerson, M. Homayoonfar, and S. C. Sharma, Inactivation of cells by heavy ion bombardment, Radiat. Res. 47, 402-425 (1971).

8. T. Saito, The nucleus-nucleus inelastic cross-sections at cosmic ray energies. J. Phys. Soc. Jpn. 38, 282 (1975).

9. H. H. Heckman, D. E. Greiner, P. J. Lindstrom, and F. S. Beiser, Fragmentation of ${ }^{14} \mathrm{~N}$ nuclei at $29 \mathrm{GeV}$ : Inclusive isotope spectra at $0^{\circ}$. Phys. Rev. Lett. 28, 926-929 (1972).

10. R. D. Evans, The Atomic Nucleus. McGraw-Hill, New York, 1955.

11. J. F. Janni, Calculations of Energy Loss, Range, Pathlength, Straggling, Multiple Scattering, and the Probability of Inelastic Nuclear Collisions for 0.1 to $100 \mathrm{MeV}$ Protons. Air Force Weapons Laboratory Technical Report No. AFWL-TR-65-150, September 1966, Air Force Systems Command, Kirtland Air Force Base, New Mexico.

12. L. C. Northcliffe and R. F. Schilling, Range and stopping-power tables for heavy ions. Nucl. Data 7, 233-463 (1970).

13. W. H. Barkas and M. J. Berger, Tables of energy losses and ranges of heavy charged particles. In Studies in Penetration of Charged Particles in Matter (U. Fano, Ed.), pp. 103-172. National Academy of Sciences-National Research Council, Washington, DC, 1964.

14. H. D. Maccabee and M. A. Ritter, Fragmentation of high-energy oxygen-ion beams in water. Radiat. Res. 60, 409-421 (1974).

15. P. Todd, Heavy-ion irradiation of cultured human cells. Radiat. Res. Suppl. 7, 196-207 (1967).

16. R. Katz and S. C. Sharma, Cellular survival in a mixed radiation environment. Int. J. Radiat. Biol. 26, 143-146 (1974).

17. R. Katz and S. C. Sharma, RBE-dose relations for neutrons and pions. Phys. Med. Biol. 20, 410 419 (1975). 